\title{
Medical Image Detection \& Privacy Management With Elliptic Curve GOPSO Cryptographic Optimization Technique on the Internet of Health Things
}

Anthony Vincent B ( $\square$ anthonyvincentphd@gmail.com )

Kristu Jayanti College

Cecil Donald A

Kristu Jayanti College

B J Hubert Shanthan

Kristu Jayanti College

Ankur Singh Bist

Graphic Era Hill University

Haider mehraj

Baba Ghulam Shah Badshah University

D. VijendraBabu

Aarupadai Veedu Institute of Technology

\section{Research Article}

Keywords: IoHT, Medical Image privacy, optimization, encryption, and decryption.

Posted Date: July 19th, 2021

DOl: https://doi.org/10.21203/rs.3.rs-714746/v1

License: (c) (i) This work is licensed under a Creative Commons Attribution 4.0 International License.

Read Full License 


\title{
Medical Image Detection \& Privacy Management with Elliptic curve GOPSO
} cryptographic optimization technique on the Internet of Health Things

\author{
${ }^{1}$ Dr. Anthony Vincent B
}

${ }^{1}$ Assistant Professor, Department of Computer Science, KristuJayanti College (Autonomous) Bangalore, Karnataka, India

anthonyvincentphd@gmail.com

\section{${ }^{2}$ Dr. Cecil Donald A}

${ }^{2}$ Assistant Professor, Department of Computer Science, KristuJayanti College (Autonomous), Bangalore, Karnataka, India

cecildonalda@gmail.com

\section{${ }^{3}$ Dr. B J Hubert Shanthan}

${ }^{3}$ Assistant Professor, Department of Computer Science, KristuJayanti College (Autonomous) Bangalore, Karnataka, India

\section{bjhubertshanthan@gmail.com}

\section{${ }^{4}$ Dr Ankur Singh Bist}

${ }^{4}$ Associate Professor, Department of Computer Science, Graphic Era Hill University, Bhimtal,India

\section{ankursinghbist6@gmail.com}

\section{${ }^{5}$ Haider mehraj}

${ }^{5}$ Department of electronics and communication engineering, Baba Ghulam Shah Badshah University, Rajouri, J\&K, India

haider_mehraj@yahoo.com

\section{${ }^{6}$ Dr.D.VijendraBabu}

${ }^{6}$ Professor \& Vice Principal, Department of Electronics \& Communication Engineering, AarupadaiVeedu Institute of Technology,Vinayaka Mission's Research Foundation, Paiyanoor-603 104,TamilNadu.India. 


\begin{abstract}
The advent of the Internet of Things (IoT) is to transform the health care sector and lead to the development of the Internet of Health Things (IoHT). This technology exceeds existing human services mechanically, financially, and socially. This paper used an advanced cryptographic framework that includes optimization strategies to look at IoHT medical image protection. The patient data kept on a cloud server which was detected and sensed from the IoHT Healthcare devices. It's critical to ensure the safety and privacy of patient clinical images in the cloud; here, an enhanced security framework for health information promotes trust. Next, we presented health care providers who could provide the full range of medical facilities for IoHT participants. In the process of encrypting/decrypting elliptical curves, the optimal key is selected using the Grasshopper Particle Swarm Optimization (GOPSO) to increase the security standard of medical images. Medical images are protected within IoHT by using this approach.The implementation results were analyzed and compared with a variety of encryption algorithms and their optimization techniques. The effectiveness of the proposed methods and the results show that the medical image is secure and prevents attacks in IoHT-based health care systems.
\end{abstract}

Keywords:IoHT, Medical Image privacy, optimization, encryption, and decryption.

\title{
I Introduction
}

IoHT creates integrated contact situations for connected devices and levels while simultaneously drawing in pragmatic and significant environments [1]. The IoT is to reach the latest technological structures with minimal human interference [2]. In the next period in digital technology, IoHT introduces 'telehealth' subsequent effort detectors and machines [3]. Conventional medical tools assign intelligence to such instruments and lead to more contact and coordination between patients and professionals from a distance [4]. Also, the protection of the IoHT integrity of the image is critically important to the development of IoHT [5]. Patient satisfaction and the provision of home care by clinical providers is another key feature in this area. In this way, various therapeutic instruments, detectors, diagnostic and imaging equipment such as intelligent devices or documents constitute the core of the IoHT [6]. In this situation, a proper framework is needed to ensure the protection and veracity of the diagnostic information of a patient that is transferred from the IoHT paradigm [7]. 
Digital media content has become increasingly relevant in people's everyday lives as interactive technologies and communication systems have advanced. It makes the storage and transmission of this data is very difficult, and it poses greater requirements on the weak functionality of the web, especially for large and moderate digital images. As limited Web bandwidth significantly limits the progress of image processing, image compression technology [8].Image segmentation, protection, and compression's main goal are to reduce the size of the image. By eliminating unnecessary or redundant information, it analyzes electronic data packets over a close to zero system. The methods of image compression could be traced to the 1948 proposal. A variety of image security transmission encoding techniques were used during this time. The genius of image detection was well documented in the early 1990s. Rather than being simply manually introduced, image attributes could be systematically developed by intelligent devices connected to the Internet.Therefore, it is critical to developing an appropriate method to ensure the confidentiality and confidentiality of the patient's clinical messages transported and extracted from the IoHT parameter. The sophisticated circuitry and sensors that connect us, each communicating valuable data [9] are surrounded by the physical objects that surround us. The transmission of this amount of data begins with the machines themselves, which should be able to cooperate securely with the IoHT process. In most countries, health care data contains highly sensitive data that should be protected by regulation under the Personal Health Information Protection Act. The effectiveness of image processing can be enhanced through the use of appropriate functionality. In previous years, image privacy and security techniques were often explicitly determined, building on previous knowledge from developers, and the number of attributes was very small. To optimize image analysis, an excellent application extraction method is required. Uncertain image characteristics could be formed using deep neural networks, and such unexpected features could also be used for image security.

We have created an encoder-decoder system model for clinical photos using IoHT, an optimum open key, and private authentication has been used in the cloud environment for image data transmission. Various methods are considered to achieve the optimum hybrid GOPSO optimization the authors of the current study have identified and analyzed the ongoing critical difficulties in strengthening IoHT protection. The rest is organized as follows: The analysis of the literature review is discussed in Chapter 2, whereas the problems of protection of medical images are discussed in Chapter 3. Chapter 4 examines the most recent proposed model, followed by the findings of Chapter 5 of the protection model. 


\section{Literature review}

A security system was developed in 2017 to provide an authentication process accessible to properties including administrators [10]. The security model updated the OpenID framework and validated a client. To prevent unauthorized access to rehabilitation equipment, an entry control system has been set up. If the confirmation is complete, the customer will receive a clearance card, also known as a Security Access Card (SAC). The productivity of the resources of the various SAC verification strategies was provisionally allocated as follows using a prototype of this system that included the estimation and precision of the detection. Malicious software attacks and confidentiality issues in the IoT were investigated in 2017 [11]. The research suggested a scientific categorization by describing and categorizing content using key criteria. Also, some reliable situational assessments have been performed to alert people to the inefficiency of IoT devices in dealing with threats. This study described and addressed a few basic open research issues (the lack of safety software upgradability, fixation of capability highlights, and a visual guarantee of billions of machines, defense, and belief).

The schema introduced that they illustrated by simulations and a prototype of complete apparatus. The result showed that the assessment of energy performance outperformed current approaches. The proposed framework reduces transmission times between smart gateways and domestic consumers by 16 percent and reduces bandwidth requirements between smart gateways and domestic consumers by 26 percent [12]. According to our study, the flexibility-induced failover inactivity is minimal, and the failover technique results in no overlap of arrangement or matching on the detector.The security framework verifies the identity of a user using the Accessible Identification Specification [13] offered in 2018. The framework ensures the authentication process and secure access to resources through IoT. After effective validation, the participant receives an approval token called an Access Control Token (ACT). It provides a collection of identifying information that allows customers to verify clinical IoT devices, facilities, and/or facilities. To prevent forgery, the SAT is cryptographically secure. Just before serving a client, a medical IoT device checks the ACT, providing a secure entry.

SecureData is a secure data collection system developed for IoT-based healthcare systems. To keep patient data secure, they simply use a covert encryption sharing strategy, which is an open-source iaas cloud computing methodology. To ensure the privacy of clinicians in the 
cloud storage layer, employ a distributed processing approach that involves several cloud service repositories [14].

The review of CoT designs and phases suggested the application of CoT in smart engineering health services in 2018 [15]. As a result, the document clarifies several relevant problems of the CoT, such as lack of institutionalization. It also emphasizes productivity performance, with careful consideration among the most important proposals available in writing. According to an analysis of all the vitality productivity strategies analyzed in this document, there remains a need to improve vitality control, particularly in terms of QoS and efficiency.For this segment, surveyed approaches given IoHT for health services and encompassing elder care, referred to as the IoHT, in terms of the most recent deliveries and products offered in the industry by the enterprise. In the same way, this research recognizes earlier mechanical developments, breaks down the challenges that need to be overcome, and provides an overview of potential trends. Despite those efforts, it is possible to assume that further research is needed to strengthen existing methods and that new ideas and developments on the Internet of Health Stuff are anticipated to address the defined challenges.

\section{Research Gap}

IoHT protection transforms the grid into a very complex structure. As such, dissatisfaction with the IoHT system could lead to more opportunities to re-establish customer support. Based on the protection technique, the data concealment approach should ensure the security of the knowledge as long as only the intended client could even see it. In other words, it is the failure of an unauthorized customer to recognize the hidden data. Accordingly, identical details will be encrypted using various security techniques such as cryptographic algorithms and the Advanced Encryption Standard (AES) algorithm.Established protective systems use cryptography, steganalysis, or a combination of the two. There is a unique, protected, and optimal means of image encryption that keeps unauthorized communications at bay [16-23]. To boost IoHT advances, anchored solutions are required to prevent the leakage of confidential communications and destructive inciting activities through peer verification and safe data exchange among IoHTcenters and repositories.

\section{Security issues in IoHT}


- Internet, smartphone, and cloud frames used to access IoHT implementations are vulnerable to a variety of threats that could significantly compromise data confidentiality.

- The IoHT physical layer contact must be protected to prevent unauthorized users to access it.

- Different threats that might ignore the identity and confidentiality of the areas could be launched on the cloud or differ IoHT depending on the sensitive network.

- The IoHT model, with its heterogeneous networks, mechanisms, and protocols, is further vulnerable to performance degradation than any other sequence.

- The IoHT system specifications will then be able to outline an appropriate method of protective measures at each level of the overall structure.

\section{Methodology}

This study suggests improving the concept of protecting the medical image of healthcare from body sensors in an IoHT environment. Medical images are saved in the IoHT based cloud storage, as seen in Figure 1, for added confidentiality, and elliptic curve cryptography is being used to encode the picture for improved security. To stabilize the image in the system, the proposed model employs an asymmetrical cryptographic method, namely the GOPSO technique.

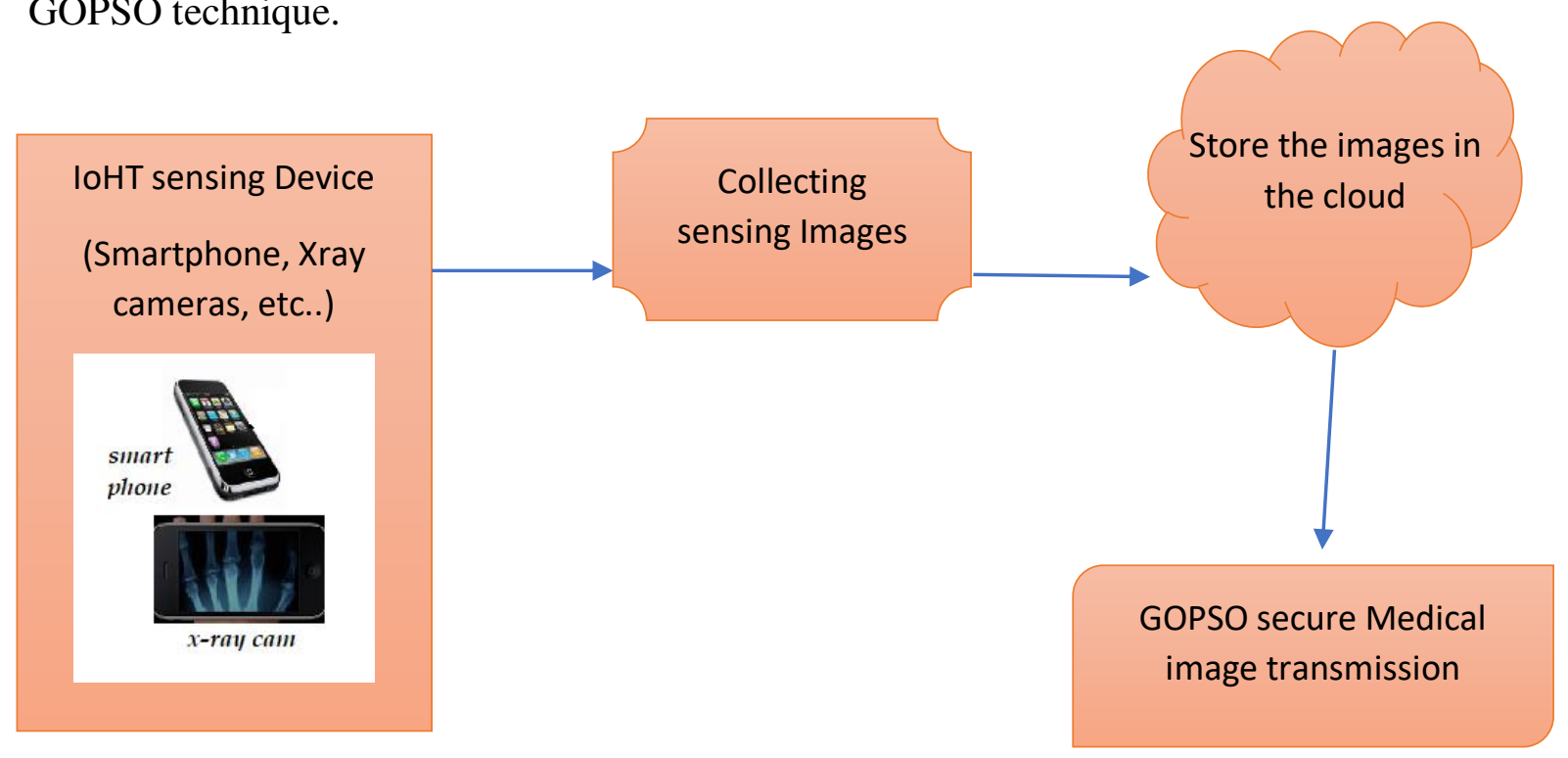

Figure 1: IoHT sensor network for cloud-based medical image storage and secure image transfer 
This cryptographic process is a highly stable encryption system provided the public and private keys are always secure. It is used to improve the reliability of the GOPSO composite optimization design offered for both keys in cryptography procedures. A procedure that can generate an encrypted image that is not restored to the original first image is what results through encryption for image security. The following sections discuss the encryption techniques and optimization method selected. Figure 2 also illustrates the concept of the proposal.

\subsection{IoHT Sensor Network}

For confidence, medical images with the cloud platform are encrypted and transmitted. IoHT technology is made up of many reduced processing detection units called sensing devices. A cloud-based framework that enables the user to monitor these devices dynamically and incrementally. Figure 1 illustrates the architecture of the IoHT sensor system. The proposed framework consists of two layers, one of which is a sensor node that detects the patient's clinical images linked to health care. The second layer gathers the observed image from the channel and then sends the data to the cloud. When a professional looks at a medical chart, he or she recommends a simple method, with drugs, and immediately sends it back to the original nucleus. In this context, the proposed model builds on its request to provide clinical disaster protection using IoHT as a key component.

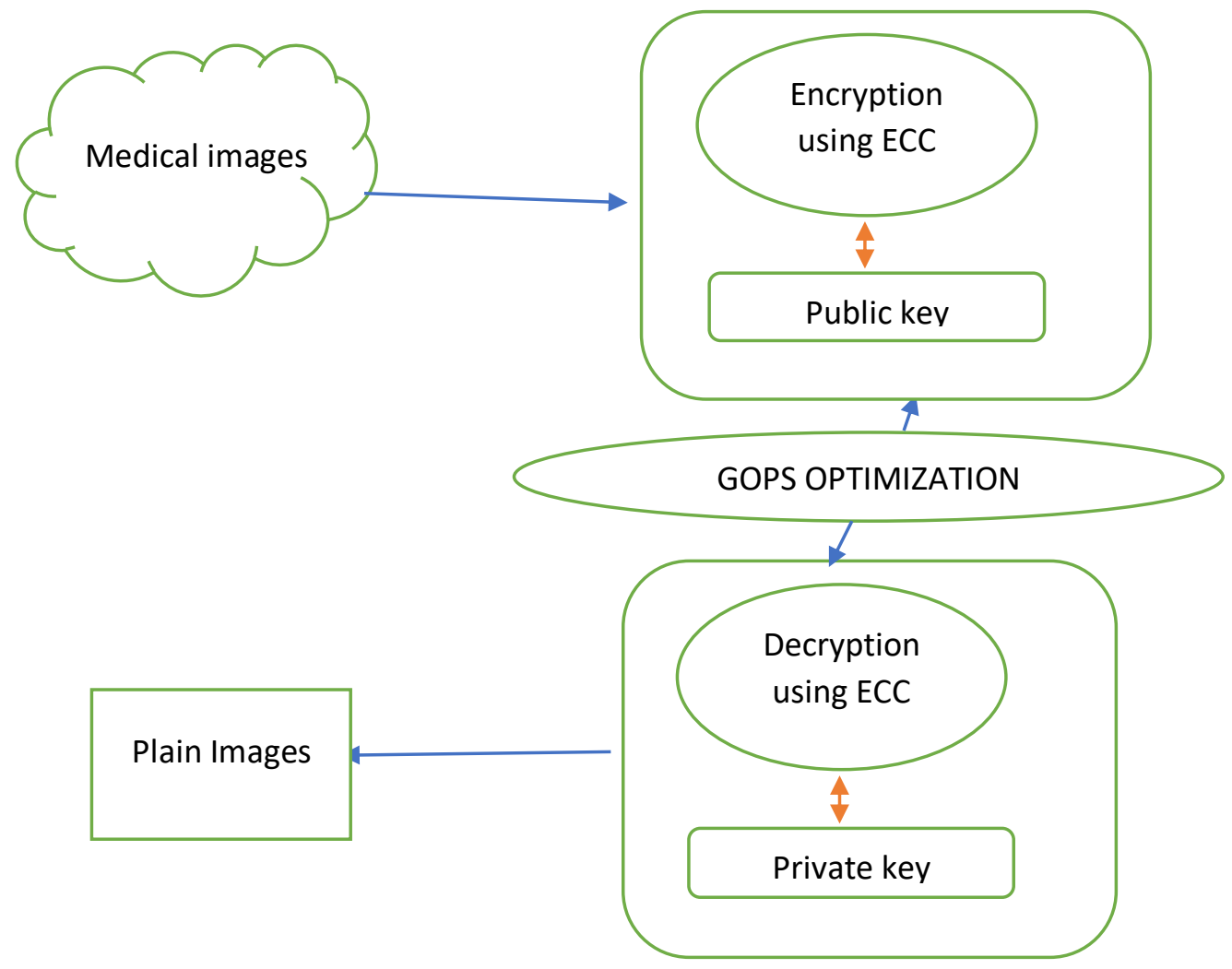


Figure 2: Graphical representation of proposed GOPSO with Elliptical curve cryptographic system

\subsection{GOPSO cryptography method}

Given the simple mathematical design of elliptical curves through finite fields, Elliptic Curve Cryptography (ECC) is a new technology for dealing with public-key encryption. It is regarded as a skill involves in image protection with a small key size, and it is extremely difficult in terms of the break period. In the medical portion, where ECC is included, this section also summarizes the IoHT medical images. This method of image encryption is illustrated in Figure 3 and has a few important phases such as key generation, encryption, and decryption. An optimization approach for the generation of public and private keys aims to improve the level of security of the IoHT system. The development of the ECC is important for practical and qualified implementation.

\section{Public and private key generation}

The functions of the ECC are divided into primary and binary phases. A rational zone with a limited number of points is selected for crypto operations. On the elliptical curve, small significant quantities of critical targets are generated by the primary phase activities, which select a primary value. The development of public and private keys, which are selected from their primary value, is critical for "ECC". The image is encrypted by using the public receiver and then the receiver's private key is used to decipher the image.

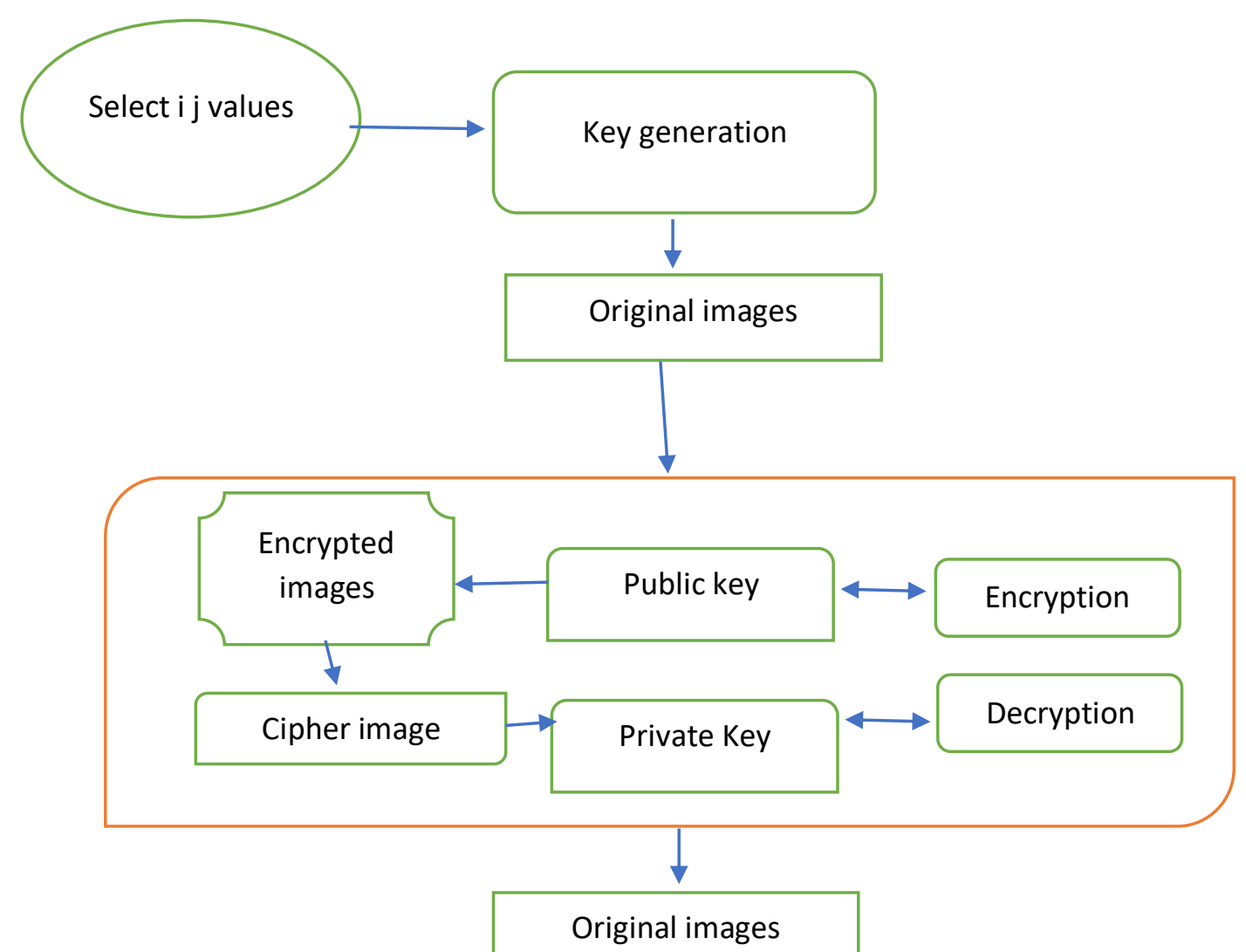


Figure 3: Encrypt and decrypt medical images with key generation.

\section{Optimizing elliptic-curve cryptography for key selection}

Math-based engineering and operations analysis all use the mathematical optimization approach, which would be the best way to select an item from a set of available choices. It is also the process of deciding the best accessibility value of an optimization problem from either a given domain or even a collection of target functions with various domain forms. There is a mixed optimization, which is a blend of GOPSO. The effective approach gets private and public keys in response to this optimization problem.

Grasshoppers work like this GO technique. The mathematical simulation uses the swarming activity of locusts, as well as the PSO algorithm, where any possible solution is treated as a particle. Each particle will have its fitness and velocity. These particles get energy from data collected on a wide variety of materials as they travel through the dimensional is sue void. In the following section, we will look at the evolution of hybrid optimization. The following steps allow the selection of keys to be optimized. First, the initialization process: (1) Each time the key strategy is introduced, the prime numbers are taken into account to create a new population size for both the optimal key selection in Equation 1.

$$
\text { Input_image }=\{\mathrm{I} 1, \mathrm{I} 2, \ldots, \mathrm{In}\}
$$

To confuse and untangle information from the medical image in IoHT, the optimal key selection method uses the "fitness operation" as the maximum key with Peak Signal-to-Noise Ratio (PSNR). The proposed hybrid methodology generates the structure by identifying the target for each arrangement. This is shown in Equation 2.

$$
\text { Fit=Max }(\text { PSNR })
$$

\section{Enhanced security optimization from GOPSO.}

The concept of establishing the integration of certain methods of such techniques is addressed in the above subsections by hybridizing Grasshopper and PSO in taking care of the optimum keys of ECC with the most severe key, i.e., PSNR. The Grasshopper's evolutionary method, combined with the instinct of natural product flies, is fully linked and precise as a new 
optimization method in finding the most restricted path, to search for food. The optimum outcome is determined by the perfect approach is illustrated in Figure 4.

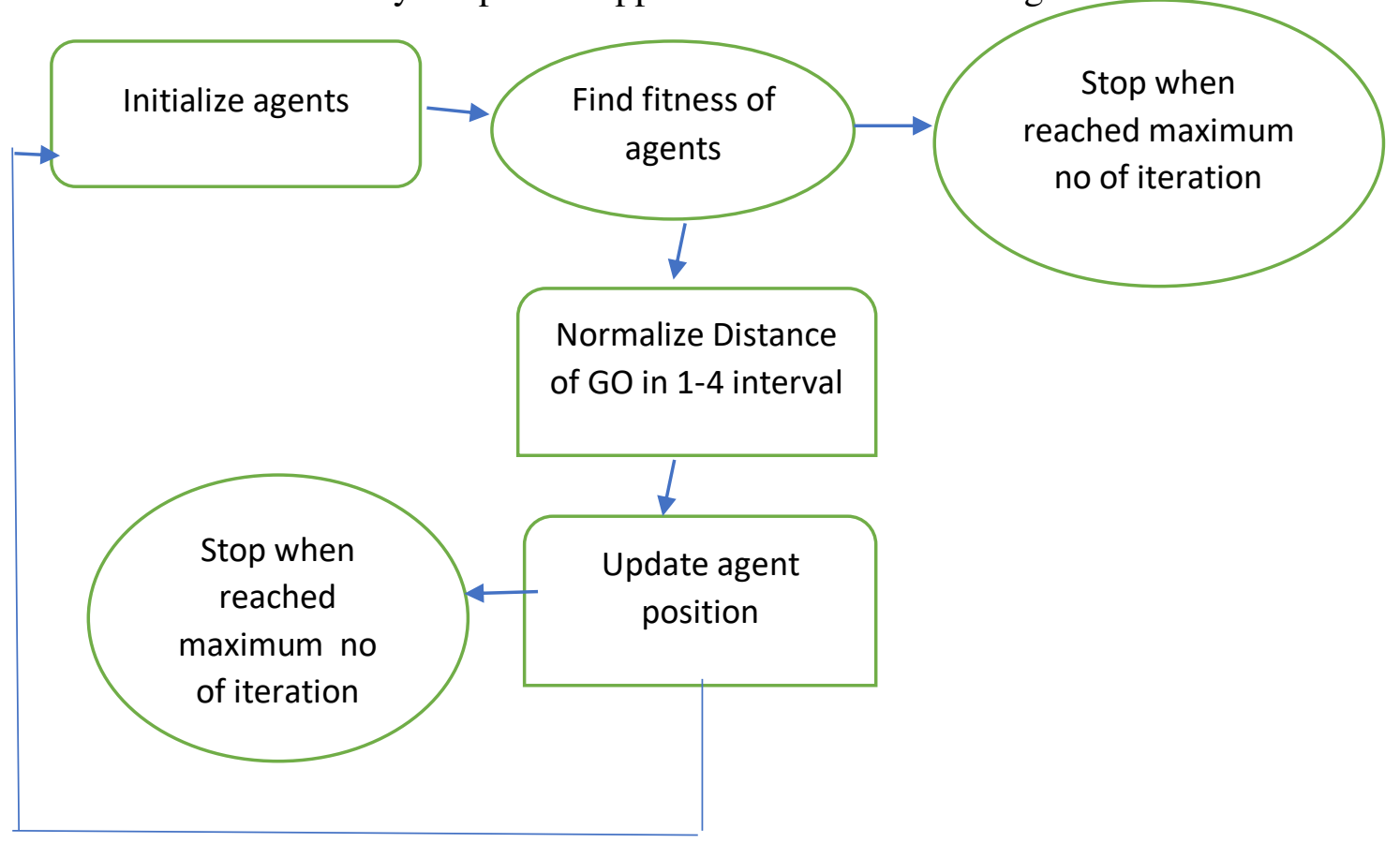

Figure 4: GOPSO hybridization process.

Grasshoppers are insects that are part of the insect category. It usually hurts agricultural productivity and agribusiness, making it a bug. The swarming behavior of these larvae and adults is a distinctive trait of the locust swarm. During the larval stage, the swarm pupa develops slowly. The main characteristic of the swarm during larval development is the slowness of the progression of the grasshopper. Equation 3 is the basic function of the grasshopper.

$$
\mathrm{Pos}_{\mathrm{i}}=\mathrm{S}_{\mathrm{i}}+\mathrm{G}_{\mathrm{i}}+\mathrm{W}_{\mathrm{i}}
$$

Where $\mathrm{S}$ is the social interaction, Gis gravity and $\mathrm{W}$ is the wind of the $\mathrm{i}^{\text {th }}$ grasshopper.

Social interaction represents the components of grasshopper growth; however, the main section begins with grasshoppers themselves. Despite the advantages of capability, it would not be able to make unique solid powers between grasshoppers separated by long distances. Distance between grasshoppers should be mapped or structured to assess this problem. It is the social relationship that has been explored in Equation 4.

$$
\mathrm{S}_{\mathrm{i}}=\sum_{j=0}^{n} s(P i j)
$$

Where Pij is the distance between the $\mathrm{i}^{\text {th }}$ and $\mathrm{j}^{\text {th }}$ grasshopper. 
The grasshopper's gravitational force is determined by Equations 6 and 7. Since nymph grasshoppers lack feathers, their growth is highly dependent on wind patterns.

$$
\begin{aligned}
& \mathrm{G}_{\mathrm{i}}=-\mathrm{GC} \\
& \mathrm{W}_{\mathrm{i}}=1 \mathrm{GD}
\end{aligned}
$$

Where GC represents the gravity constant represents the constant drift, and GD represents the unit vector in the whole wind direction. To solve the optimization problem, a probabilistic model should be successfully exploited to determine the actual approximation of the optimum solution. The function is in addition to the preceding model. This saves the optimum response to each measuring cycle. The mathematical model illustrated above must be equipped with specific parameters to illustrate discovery and optimization at various stages of optimization.

\section{PSO and velocity updating process}

PSO is a probabilistic global optimization technique derived from swarm knowledge and based on the analysis of the production activity of winged creatures and fish. A fitness function determines the importance of each molecule's main purpose. To begin, the particles are randomly inserted with velocity and position. This PSO demonstrates important parameters such as global finest $(\mathrm{gb})$ and particle finest $(\mathrm{pb})$. The velocity and new updated solutions are evaluated in the Elliptic curve for optimal main selection. The following equation is used for adjusting velocity and position.

$$
\begin{aligned}
& \mathrm{VE}_{(\mathrm{t}+1)}=\mathrm{VE}_{\mathrm{i}(\mathrm{t})}+\mathrm{w} 1 * \mathrm{u} *\left(\mathrm{pb}_{(\mathrm{t})}\right)-\mathrm{x}_{\mathrm{i}(\mathrm{t}))}+\mathrm{w} 2 * \mathrm{u}^{*}\left(\mathrm{gb}_{(\mathrm{t})}-\mathrm{x}_{\mathrm{i}(\mathrm{t}))}\right) \\
& \mathrm{x}_{\mathrm{i}(\mathrm{t}+1)}=\mathrm{u}_{\mathrm{i}(\mathrm{t})}+\mathrm{VE}_{(\mathrm{t}+1)}
\end{aligned}
$$

VEi is the particle velocity; Ui is the current particle; w1 and w2 are learning variables, with the weight of w1 and w2 respectively. The ith particles location is organized by the circumstance of global finest arrangement and position of particle finest arrangement, according to the refreshing technique laid out on conditions as in Equations 9 and 10. The approach is followed to the point where, in terms of this refreshing design, the achievement of the solution through widespread fitness esteem locates the optimal key to establish a medical image in IoHT.

\section{Image encryption and decryption phases}


Encryption is a method of converting images or data in a manner that allows them to be authorized. Whenever the sender's image data was spoken with the curve, the curve position was found, which was used to transform the plain images into ciphered pictures, where the public keys were selected.

Decryption is the polar opposite of encoding, i.e., the process of transforming an encoded material into its amazing plain picture. Following this process, the confused data or image is displayed, while the portion of the image is damaged and preventing illegal access. By using an elliptical curve, the image is decoded by converting the cipher image to a plain image with the private key.

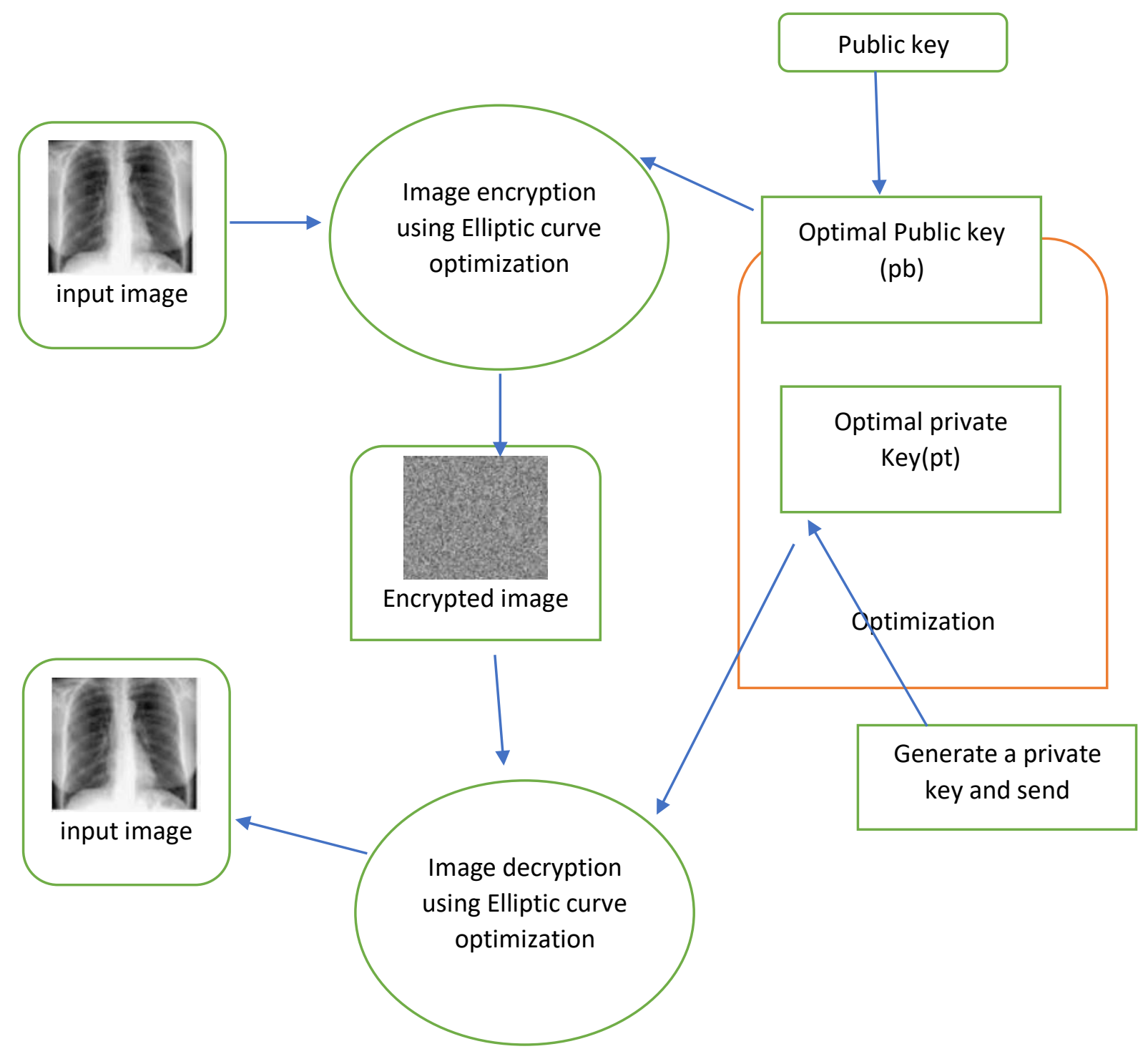

Figure 5: Optimized Elliptic curve-based encryption and decryption process

For reliable and efficient coordination, the encoded picture is transmitted as an encrypted file. It keeps an opponent from engaging in malicious activities while enhancing classification. 
ECC techniques are used to enhance the security criteria for the IoHT and cloud model. Figure 5 depicts the optimal key-based medical image encryption and decryption process.

\section{Implementation}

Let us take the input for information as ' $\mathrm{a}$ ' in Equation 11,Elliptic curve values as $\mathrm{k}, 1$ and prime number $\mathrm{z}$,

$$
\mathrm{EC}=\mathrm{a}^{3}+\mathrm{ka}+\mathrm{l}: \mathrm{k}=\mathrm{l}=2
$$

Compute the curve function is expressed in Equation 12,

$$
\mathrm{k}=\bmod (\mathrm{EC}, \mathrm{Bm}) \text { and } 1=\bmod (\mathrm{B}(\mathrm{J})) 2, \mathrm{BQ})
$$

Generate a key using the following steps

The integer values for private key Ptk are chosen

Generate Public key Pbk =Ptk *T

Where $\mathrm{T}$ is the random value $(1, \ldots,(\mathrm{n}-1))$

Compute optimal public and private keys by utilizing optimization.

Update GOPSO solution

Repeat until optimal OPtk, OPbk is generated.

\section{Encryption and decryption process:}

Let us consider that the sender is transmitting the receiver a photo. It encodes an elliptic group position over to a plain image. The experiment transforms the ciphered photos as R1 and $\mathrm{R} 2$ to select random integer variables o from the range of $\mathrm{i}$ and $\mathrm{i}-1$ is calculated in Equation 13.

$$
\begin{array}{r}
\mathrm{R} 1=\mathrm{o}^{* \mathrm{c}} \\
\mathrm{R} 2=1+0 * \mathrm{OPbk}
\end{array}
$$

Decode the image with a private key is shown in Equation 14.

$$
\begin{array}{r}
\text { Rplain }=\text { OPtk } * \mathrm{R} 1 \\
\text { Plain image }=\mathrm{R} 2-\mathrm{OPtk}-\mathrm{R} 1
\end{array}
$$


The receiver finally decodes the image with the private key by computing the first point of $\mathrm{R} 1$ and $\mathrm{R} 2$ to get the plain image.

\section{Result Analysis}

Our proposed IoHT privacy model has been implemented in Net beans using the Java JDK 1.7.0 language on the Windows system. With Intel $(\mathrm{R})$ Core i5 processors operating at 1.6GHz and 4GB of RAM. And the images produced by IoHT sensors are stored in the cloud environment.

Table 1: execution performance measurement

\begin{tabular}{|c|c|}
\hline PSNR & MSE(Mean square error) \\
\hline PSNR $=10^{*} \log 10(\mathrm{M} 2$ & $\mathrm{MSE}=1 / \mathrm{n} \sum_{\mathrm{i}}^{\mathrm{n}}(\mathrm{Ai}-\mathrm{Bi}) \quad 2$ \\
$/ \mathrm{MSE})$ & \\
\hline
\end{tabular}

The brain and lung are the sample diagnostic photos from the cloud. The private image was analyzed both before and after it was sent and obtained by the intended recipient.

Table 2: Result of proposed GoPSO optimization of image

\begin{tabular}{|c|c|c|c|}
\hline Images & $\begin{array}{c}\text { Encrypted } \\
\text { image }\end{array}$ & PSNR & MSE \\
\hline & & 51.21 & 0.10 \\
\hline & & & \\
\hline
\end{tabular}




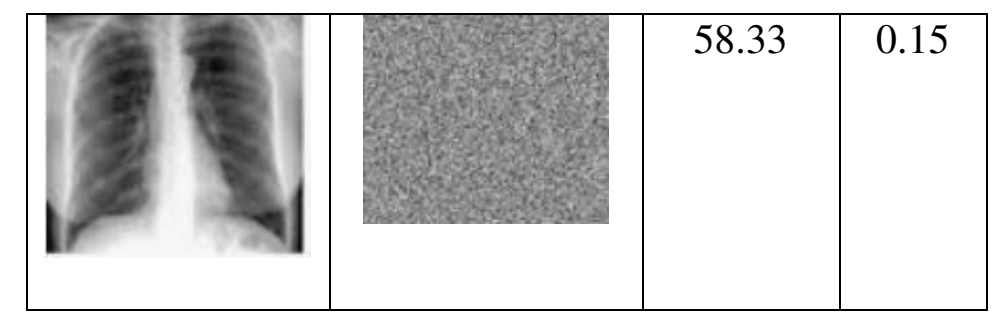

This is performed to verify that the first cover will have less noise inside after the private image has been hidden. Standard elliptic curve and other encryption techniques, such as AES and DES, were compared to this proposed system. These measures shown in Table 1 such as PSNR and MSE, evaluate the proportions between the first image and the encrypted images, while the computational analysis is discussed in the following segments.

Table 2 shows the impact of the proposed Elliptic curve-based GOPSO method based on PSNR, MSE, and the calendar for various images.

The convergence of the PSNR fitness operation is illustrated in Figure 6, which is different from the traditional optimization Particle swarm optimization and GOPSO.

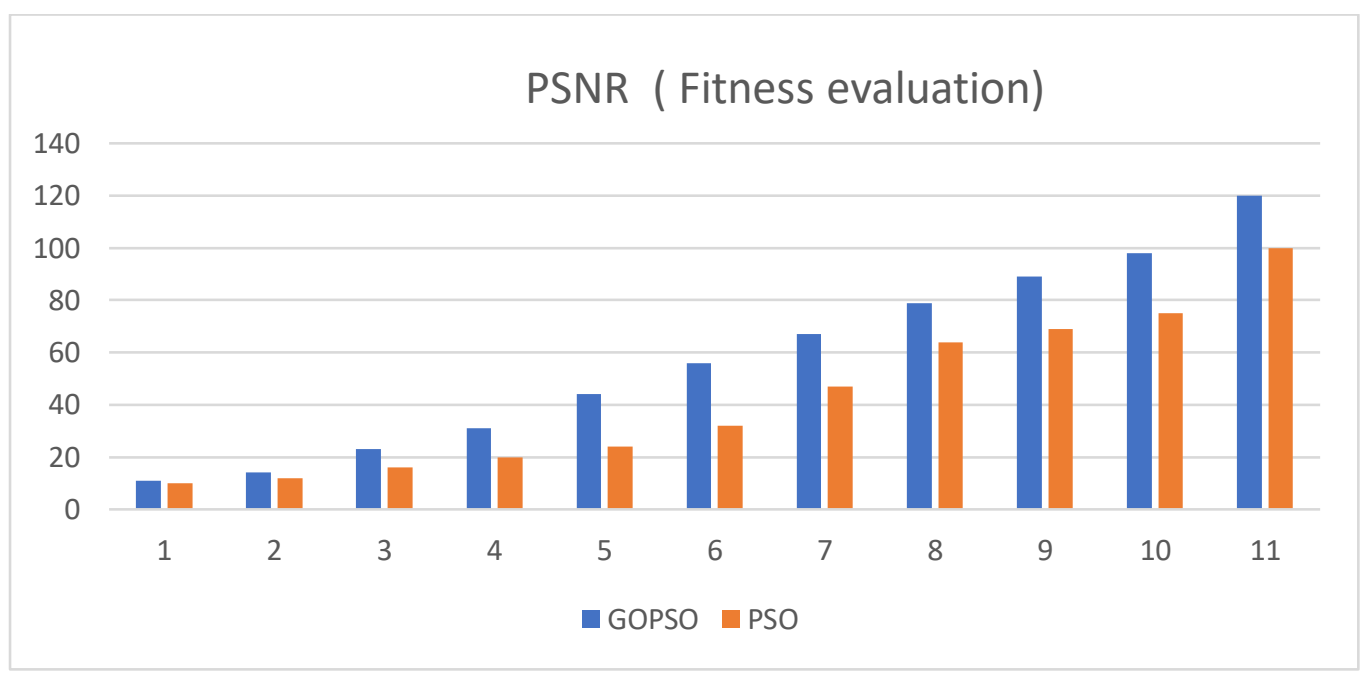

Figure 6: Fitness evaluation

Table 3 describes the intended intrusion in the proposed system. In which two attacks are considered salts and are linked to a sample of three medical input images to determine security execution. However, the criteria are: first, attack the watermarked image with some attack, then recover the method of protection.

Table 3: Proposed system Attack based result 


\begin{tabular}{|c|c|c|c|}
\hline Images & Attack & PSNR & MSE \\
\hline 1 & & 58.55 & 0.9 \\
\hline 2 & & & \\
\hline & & & \\
\hline & & & \\
\hline & & & \\
\hline & & & \\
\hline
\end{tabular}

The PSNR of the resulting db values of salt sound is different levels which have produced different results. And at this point, the execution analysis variables are less for the attacks applied in the picture compared to the proposed work. Tables 4 and 5 show the images obtained by protecting medical images with and without attack. When multiple assaults are linked to different images, the PSNR and MSE estimate of the "no-assault image" is the best option. As the noise density increases, the PSNR will change and be visually represented.

Table 4: Attack vs without Attack (PSNR)

\begin{tabular}{|r|r|}
\hline \multicolumn{2}{|c|}{ PSNR } \\
\hline $\begin{array}{r}\text { Image with } \\
\text { attack }\end{array}$ & \multicolumn{2}{l|}{$\begin{array}{l}\text { Image without } \\
\text { attack }\end{array}$} \\
\hline 53.2 & 58.55 \\
\hline 40.5 & 59.34 \\
\hline 60.5 & 61.89 \\
\hline
\end{tabular}

Table 5:Attack vs without attack (MSE)

\begin{tabular}{|l|l|}
\hline \multicolumn{2}{|c|}{ MSE } \\
\hline $\begin{array}{l}\text { Image with } \\
\text { attack }\end{array}$ & $\begin{array}{l}\text { Image without } \\
\text { attack }\end{array}$ \\
\hline 0.9 & 0.10 \\
\hline
\end{tabular}




\begin{tabular}{|r|r|}
\hline 0.11 & 0.12 \\
\hline 0.12 & 0.15 \\
\hline
\end{tabular}

The above experimental results were represented in graphical form for better understandings in the following Figure 7 (a) and (b).

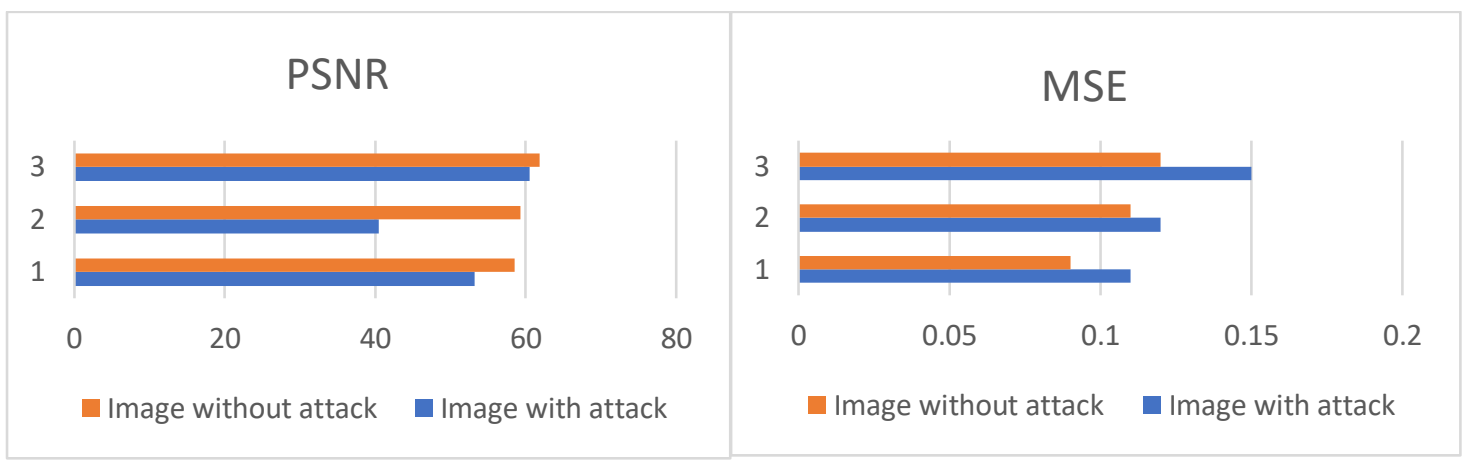

Figure 7(a \& b) PSNR and MSE attack vs without attack images

\section{Conclusion}

We analyzedIoT-based services and applications in this research study and introduced a new medical image secure scheme to provide high image protection and guarantee the confidentiality of patients' private information. The results indicate that the proposed algorithm would maximize the number of participants while improving the reliability of cloud-based data confidentiality. The study also suggested a hybrid strategy based on a cryptographic algorithm to enhance IoHT. The ECC with GOPSO is a proposed model that uses clinical image encryption and decoding to obtain high security.Because there is less financial uncertainty, the technology requires less storage. We used critical measures such as PSNR and MSE to demonstrate the current work with different parameters, which suggested controlling image privacy in all studies. It is clear that the procedure is sufficiently secure because it has resulted in high impalpability; however, it should be further studied to increase the level of protection. Both encrypting/decrypting processes require less time with the proposed method.In future studies, we will explore how techniques are applied in-depth with different steps using advanced cryptographic approaches and hybrid optimization methodology. This would apply to a variety of cloud-based data protection applications for threats and attacks.

\section{Conflict of interest:}

There is no conflict of interest. 


\section{Funding:}

There is no funding information.

\section{Availability data and material:}

There is no Availability data and material.

\section{Code Availability:}

There is no code availability.

\section{Author's contribution:}

There is no Author's contribution.

\section{References}

1. Gaber T, Abdelwahab S, Elhoseny M, Hassanien AE (2018) Trust-based secure clustering in WSN-based intelligent transportation systems. Comp Netw. https://doi.org/10.1016/j.comnet. 2018.09.015

2. Islam SR, Kwak D, Kabir MH, Hossain M, Kwak KS (2015) The internet of things for health care: a comprehensive survey. IEEE Access 3:678-708

3. Thibaud M, Chi H, Zhou W, Piramuthu S (2018) Internet of things (IoT) in a highrisk environment, health, and safety (EHS) industries: a comprehensive review. Decis Support Syst 108:79-95

4. Pizzolante R, Castiglione A, Carpentieri B, De Santis A, Palmieri F, Castiglione A (2017) On the protection of consumer genomic data on the internet of living things. ComputSecur 74:384-400

5. Tankard C (2015) The security issues of the internet of things. Comput Fraud Secur 2015(9):11-14

6. Rahmani AM, Gia TN, Negash B, Anzanpour A, Azimi I, Jiang M, Liljeberg P (2018) Exploiting smart e-health gateways at the edge of healthcare internet-of-things: a fog computing approach. Future GenerComputSyst 78:641-658

7. Kothmayr T, Schmitt C, Hu W, Bru “nig M, Carle G (2013) DTLS based security and two-way authentication for the internet of things. Ad Hoc Netw 11(8):2710-2723

8. G. Litjens, T. Kooi, B.E. Bejnordi, et al., A survey on deep learning in medical image analysis, Med. Image Anal. 42 (2017) 60-88.

9. Z. Zeng, W. Xie, Y. Zhang, et al., RIC-UNet: An improved neural network based on UNet for nuclei segmentation in histology images, IEEE Access 7 (99) (2019) 2142021428.

10. Hossain M, Islam SR, Ali F, Kwak KS, Hasan R (2017) An internet of things-based health prescription assistant and its security system design. Future GenerComputSyst $1-26$.

11. Yaqoob I, Ahmed E, urRehman MH, Ahmed AIA, Al-garadi MA, Imran M, Guizani $M$ (2017) The rise of ransomware and emerging security challenges in the internet of things. ComputNetw 129:444-458. 
12. Elsir, A., Elsier, O., Abdurrahman, A., \&Mubarakali, A. (2019). Privacy Preservation in Big Data with Data Scalability and Efficiency Using Efficient and Secure Data Balanced Scheduling Algorithm.

13. Ramakrishnan, J., Mavaluru, D., Sakthivel, R. S., Alqahtani, A. S., Mubarakali, A., \&Retnadhas, M. (2020). Brain-computer interface for amyotrophic lateral sclerosis patients using deep learning network. NEURAL COMPUTING \& APPLICATIONS.

14. Loganathan, J., Janakiraman, S., \&Latchoumi, T. P. (2017). A Novel Architecture for Next Generation Cellular Network Using Opportunistic Spectrum Access Scheme. Journal of Advanced Research in Dynamical and Control Systems,(12), 1388-1400.

15. Loganathan, J., Janakiraman, S., Latchoumi, T. P., \&Shanthoshini, B. (2017). Dynamic Virtual Server For Optimized Web Service Interaction. International Journal of Pure and Applied Mathematics, 117(19), 371-377.

16. Andrew J, \&Karthikeyan J. (2019). Privacy-Preserving Internet of Things: Techniques and Applications. International Journal of Engineering and Advanced Technology (IJEAT), 8(6), 3229-3234. https://doi.org/10.35940/ijeat.F8830.088619

17. Latchoumi, T. P., Ezhilarasi, T. P., \&Balamurugan, K. (2019). Bio-inspired weighed quantum particle swarm optimization and smooth support vector machine ensembles for identification of abnormalities in medical data. SN Applied Sciences, 1(10), 1-10.

18. Battula, B., Anusha, V., Praveen, N., Shankar, G., \&Latchoumi, T. P. (2020). Prediction of Vehicle Safety System Using Internet of Things. Journal of Green Engineering, 10(4), 1786-1798.

19. Al, M., Chanyaswad, T., \& Kung, S.-Y. (2018). Multi-Kernel, Deep Neural Network and Hybrid Models for Privacy-Preserving Machine Learning. 2018 IEEE International Conference on Acoustics, Speech and Signal Processing (ICASSP), 2891-2895. https://doi.org/10.1109/ICASSP.2018.8462336.

20. Ezhilarasi, T. P., Dilip, G., Latchoumi, T. P., \&Balamurugan, K. (2020). UIP-A Smart Web Application to Manage Network Environments. In Proceedings of the Third International Conference on Computational Intelligence and Informatics (pp. 97-108). Springer, Singapore.

21. M. Varma, A. Zisserman, A statistical approach to material classification using image patch exemplars, IEEE Trans. Pattern Anal. Mach. Intell. 31 (11) (2009) 2032-2047.

22. J. Long, E. Shelhamer, T. Darrell, Fully convolutional networks for semantic segmentation, IEEE Trans. Pattern Anal. Mach. Intell. 39

23. (2014) 640-651. [8] J. Dolz, C. Desrosiers, I.B. Ayed, 3D fully convolutional networks for subcortical segmentation in MRI: A large-scale study, Neuroimage (2016) 170:S1053811917303324. 